\title{
Modularizing Contexted Constraints
}

\author{
John Griffith* \\ Seminar für Sprachwissenschaft: \\ Universität 'Tïbingen \\ K1. Wilhelmstr. 113, \\ D-72074 Tübingen, Germany \\ griffith@sfs.nphil.uni-tuebingen.de
}

\begin{abstract}
This paper describes a method for compiling a constraint-based grammar into a potentially more efficient form for processing. This method takes dependent, disjunctions within a constraint formula and factors them into non-interacting groups whonever possible by detcrmining their independence. When a group of dependent disjunctions is split into smaller groups, an exponential amount of redundant information is reduced. At runtime, this means that an exponential amount, of processing can be saved as well. Since the performance of an algorithm for processing constraints with dependent disjunctions is highly determined by its input, the transformation presented in this paper should prove beneficial for all such algorithms.
\end{abstract}

\section{Introduction}

There are two facts that conspire to make the treatment of disjunction an important consideration when building a natural language processing (NLP) system. The first fact is that natural languages are full of ambiguities, and in a grammar many of these ambiguities are described by disjunctions. The second fact is that the introduction of disjunction into a grammar causes processing time to increase exponentially in the number of disjuncts. This means that a ncarly lincar-time operation, such as unification of purely conjunctive feature structures, bccomes an exponential-time problem as soon as disjunctions are included. ${ }^{1}$ Since disjunction is unlikely to dis-

\footnotetext{
* This work was sponsored by Teilprojekt B4 "From Constraints to Rules: Compilation of irpsa" of the Sonderforschungsbereich 340 of the Doutsche Forschungsgemeinschaft. I would also like to thank Dale Gerdemann and Guido Minnen for helpful comments on the ideas presented here. All remaining errors are of course my own.

${ }^{1}$ Assuming $P \neq N I$.
}

appear from natural language grammars, controlling its form can save exponential amounts of time.

This paper introduces an efficient normal form for processing dependent disjunctive constraints and an opcration for compilation into this normal form. This operation, modularization, can reduce exponential amounts of redundant information in a grammar and can consequently save corresponding amounts of processing time. While this operation is general enough to be applied to a wide varicty of constraint systems, it was originally designed to optimize processing of dependent disjunctions in feature structure-based grammars. In particular, modular feature structures are more efliciont for unification than non-modular ones. Since in many current NLP systems, a significant amount of time is spent performing unification, optimizing feature structures for unification should increase the performance of these systems.

Many algorithms for efficient mification of feature structures with dependent disjunctions have been proposed (Maxwell and Kaplan, 1989; Eisele and Dörre, 1990; Gerdemann, 1991; Strömbärck, 1992; Griflith, 1996). However, all of these algorithms suffer from a common problem: their performance is highly determined by their inputs. All of these algorithms will perform at their bost when their dependent disjunctions interact as litthe as possible, but if all of the disjunctions interact, then these algorithms may perform redundant computations. The noed for efficient inputs has been noted in the literature ${ }^{2}$ but there have been few attempts to automatically optimize grammars for disjunctive unification algorithms.

The modularization algorithm presented in this paper takes existing dependent disjunctions and splits thom into independent groups by determining which disjunctions really interact. Independent groups of disjunctions can be processed separately during unification rather than having to try every combination of one group with every combination of every other group.

This paper is organized as follows: Section 2 gives an informal introduction to dependent dis-

\footnotetext{
${ }^{2}$ Cf. (Maxwell and Kaplan, 1991) for instance.
} 
junctions and shows how redundant interactions between groups of disjunctions can be reduced. Section 3 shows how normal disjunctions cin be replaced by contexted constraints. Section 4 then shows how these contexted constraints can encode dependent disjunctions. Section 5 presentsis the modulariation algorithm for contexted constraints. However, even though this algorithm is a compile-time operation, it, itsolf has exponential complexity, so making it more officient should also be a concent. $\Lambda$ theorem will then be presented in section 6 that permits an exponential prart of the modularization algorithn to bo replaced by combinatorial analysis.

\section{Dependent disjunctions}

Dependent, disjunctions are like normal disjunctions except that every disjunction has a name, and the disjuncts of disjunctions with the same? name must be chosen in sync. Lior example, $\left\langle{ }_{d} \phi, \phi^{\prime}, \phi^{\prime \prime}\right\rangle \wedge\left\langle{ }_{d} \psi, \psi^{\prime}, \psi^{\prime \prime}\right\rangle$ is a conjunction of two dependent disjunctions with the same name, $d$. What this means is that if the second disjunct in the first disjunction, $\phi^{\prime}$, is chosen, then the second disjunct of the other alisjunction, $\psi^{\prime}$, must. be chosen as well. (Note that what kind of constraints the ds and $\psi$ s are is not, important here.) The computiational reason for using dependent disjunctions over nomal disjunctions is that dependent disjunctions allow for more compact and efficient structures. 'This is particularly true when dependent disjunctions are embedded inside of feature structures. This is because in that case disjunctions can be kept local in a directed graph structure thess saving redundant foature pathis.

We say that disjunctions with the same name are in the same group. One distinguishing fea ture of a group of disjunctions is that cach disjunction must have the same number of disjuncts. This is essentially where rechundant interactions originate. For instance, in $\left\langle a \phi, \phi, \phi^{\prime}, \phi^{\prime}\right\rangle \wedge$ $\left\langle{ } \psi \psi, \psi^{\prime}, \psi, \psi^{\prime}\right\rangle$ each disjumction has fonr disjumets, but really only two values. But more importiantly, no matice what value of the lirst clisjunction is chosen $\left(\phi\right.$ or $\left.\phi^{\prime}\right)$ the same values are pos. sible for the second $\left(\psi\right.$ or $\left.\psi^{\prime}\right)$. In other words, these disjunctions are actually independent from one another, and can be put into diflerent groups: $\left\langle d^{\prime} \phi, \phi^{\prime}\right\rangle \wedge\left\langle d^{\prime \prime} \psi, \psi^{\prime}\right\rangle$. 'This is the process of modularization which will be formalized in section 5 .

One might be tempted to think that moclularization is unnecessary since grammar writers are nulikely to write dependent disjunctions which contain indepondent parts. Jlowever, grammar writers may not be the only source of dependent. disjunctions. Many gramman processing systems use high-level descriptions which are then transformed into more explicit lower-level graumars. This transformation process may very well introduce large numbers of dependent disjunctions with exactly this property.

One example of where this can happen is in the compilation of lexical rules in (Meners and Minmen, 1995). In this paper, Meurers and Minnen describe a compiler which translates a set of HISS lexical rules and their interaction into definite relations used to constrain lexical ontries. In (Meurers and Minnon, 1996), thoy show how an off-line compilation technique called constraint propagation can be used to improve the definite clause encoding produced by their compiler to al w. low for mone efliecient processing. 'The use of de pendent disjunctions provides an attractive alter. mative to the constraint propagation approach by spexilying all the information associated with a lexical entry dincetly as a single dependent fort ture stutudure rather than hidelen in a set of definite clanses." Consicler the AVM below:

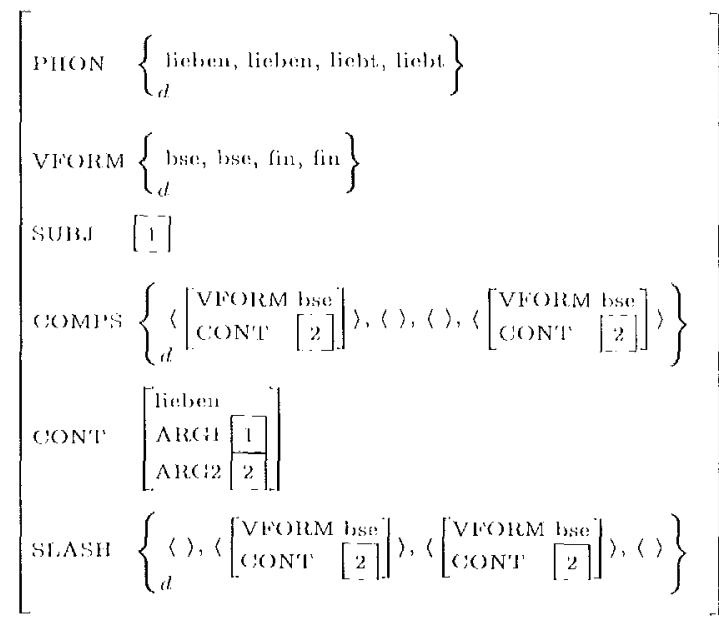

This complex lexical entiry represents the basc lex ical entry for the German verb liceben, "to love", and the three lexical entries that can be derived from it givon tho lexical rules presented in (Mourers and Minmen, 1996). The differences between these lexical entries are encoded by the dependent disjunctions all of which are in the same? gronp, a. The first alisjunct in ach disjunction corresponds to the base form, the socond corresponds to the applicalion of the Complement Fxtraction laxical Rule, the thind corresponds to the appliaation of the linitivization Lexical Rule, and the last, corresponds to the application of both rules. ${ }^{4}$ Modularization can be used to make this feature structure even more efficient by splitting the group $d$ into two new groups $d_{1}$ and $d_{2}$ as shown below.

\footnotetext{
${ }^{3}$ In the case of infinite lexica, definite clanses are still necessary to encode recursive information.

${ }^{1}$ l'hese lexical rules are simplified versions of those presented in ('ollard and Sag, 1994).
} 


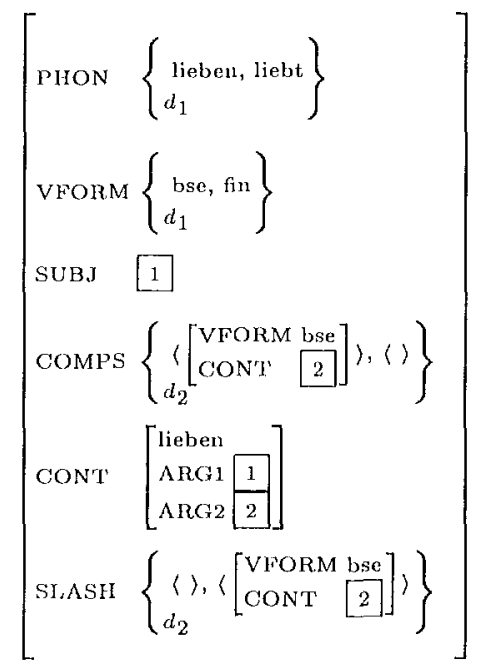

Another example of where modularization might prove useful is in the treatment of typed feature structures presented in (Gerdemann and King, 1994). Their approach produces a set of feature structures from a satisfiability algorithm such that all of the feature structures have the same shape but the nodes may be labeled by difforent types. They then collapse this set down to a single feature structure where nodes are labeled with dependent disjunctions of types. Many of the groups of disjunctions in their feature structures can be made more efficient via modularization.

A final example is in the compaction algorithm for feature structures, presented in (Griffith, 1995). Compaction is another operation designed to optimize feature structures for unification. It takes a disjunction of feature structures, transforms them into a single feature structure with dependent disjunctions, and then pushes the disjunctions down in the structure as far as possible. The result is a large number of dependent disjunctions in the same group. Many of these can probably be split into new independent groups.

\section{Contexted constraints}

Maxwell and Kaplan (1989) showed how a disjunction of constraints could be replaced by an cqui-satisfiable conjunction of contexted constraints as in lemma 1 below. 5

\section{Lemma 1 (Contexted Constraints)}

$\phi_{1} \vee \phi_{2}$ is satisfiable iff $\left(p \rightarrow \phi_{1}\right) \wedge\left(\bar{p} \rightarrow \phi_{2}\right)$ is satisfiable, where $p$ is a new propositional variable.

Disjunctions are replaced by conjunctions of implications from contexts (propositional formulae) to the base constraints (ie. $\phi_{1}$ and $\phi_{2}$ ). The nature of the base constraints is irrelevant as long as there is a satisfaction algorithm for them. The key insight, is that solving disjunctions of the base constraints is no longer necessary since they are purely conjunctive.

\footnotetext{
${ }^{5}$ For a proof see (Maxwell and Kaplan, 1989).
}

Maxwell and Kaplan's goal in doing this was to have an efficient method for solving disjunctive constraints. The goal in this paper is compiling disjunctive constraints into more efficient ones for future solution. To this end a somewhat different notion of contexted constraint will be used as show in lemma 2.

\section{Lemma 2 (Alternative-Case Form)}

$\phi_{1} \vee \phi_{2}$ is satisfiable iff $\left(a_{1} \rightarrow \phi_{1}\right) \wedge\left(a_{2} \rightarrow \phi_{2}\right) \wedge$ $\left(a_{1} \vee a_{2}\right)$ is satisfiable, where $a_{1}$ and $a_{2}$ are new propositional variables.

We can see that this formulation is nearly equivalent to Maxwell and Kaplan's by substituting $p$ for $a_{1}$ and $\bar{p}$ for $a_{2}$. To make the formulation completely equivalent, we would need to enforce the uniqueness of a solution by conjoining $\bar{a}_{1} \vee \bar{a}_{2}$. However, this is unnecessary since we want to permit both solutions to be simultancously true. The reason for using the modified version of contexted constraints in lemma 2 is that we can separate the representation of disjunctions into a conjunction of the values that the disjuncts can have, called the alternatives, and the way in which the we can choose the values, called the cases. The alternatives are the conjunction $\left(a_{1} \rightarrow \phi_{1}\right) \wedge\left(a_{2} \rightarrow \phi_{2}\right)$ and the cases are the disjunction $\left(a_{1} \vee a_{2}\right)$.

While we could use repeated applications of lemma 2 to turn a disjunction of $n$ disjuncts into an alternative-case form, it will simplify the exposition to have a more general way of doing this, as shown in lemma 3 .

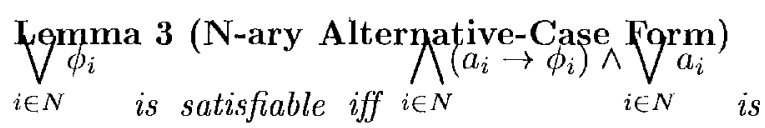
satisfiable, where each $a_{i}$ is a new propositional $\operatorname{variabl}^{6}\left(a_{i} \rightarrow \phi_{i}\right)$

Here $i \in N \quad$ are the alternatives and $i \in N$ are the cases. So for example, $\phi_{1} \vee \phi_{2} \vee \phi_{3} \vee \phi_{4}$ is satisfiable just in case $\left(a_{1} \rightarrow \phi_{1}\right) \wedge\left(a_{2} \rightarrow \phi_{2}\right) \wedge$ $\left(a_{3} \rightarrow \phi_{3}\right) \wedge\left(a_{4} \rightarrow \phi_{4}\right) \wedge\left(a_{1} \vee a_{2} \vee a_{3} \vee a_{4}\right)$ is satisfiable.

\section{Dependent disjunctions as contexted constraints}

The usefulness of the alternative-case form only becomes apparent when considering dependent disjunctions. Dependent disjunctions can be represented by alternative-case forms as shown in definition 1 below.

\section{Definition 1 (Dependency Group)}

$A$ dependency group is a conjunction of dependent disjunctions with the same name, $d$, where each

$$
\begin{aligned}
& \bigvee_{\sigma_{i \in N}} \bigwedge_{i} \phi_{i} \\
& \text { and formulae } \phi_{i}, \text { respectively, where each } i \text { is a member }
\end{aligned}
$$
of the set of indices, $N$. 
disjunction is an alternative-case form such that there is one alternative for every disjunct of every disjunction in the group, and there is one case for each disjunct in the group which is a conjunction of the alternative variables for that disjunct

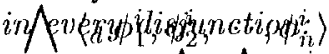

$$
\bigwedge_{i \in M} \bigwedge_{i \in M} \bigwedge_{j \in N}\left(a_{j}^{i} \rightarrow \phi_{j}^{i}\right) \bigvee_{\wedge j \in N} \bigwedge_{i \in M} a_{j}^{i}
$$

where each $a_{j}^{i}$ is a new propositional variable and $N=\{1,2, \ldots, n\}$.

So the dependent disjunction $\left\langle{ }_{d} \phi, \phi, \phi^{\prime}\right\rangle \wedge$ $\left\langle d \psi, \psi^{\prime}, \psi^{\prime}\right\rangle$ is the alternative-case form with alternatives $\left(a_{1}^{1} \rightarrow \phi\right) \wedge\left(a_{2}^{1} \rightarrow \phi\right) \wedge\left(a_{3}^{1} \rightarrow \phi^{\prime}\right) \wedge$ $\left(a_{1}^{2} \rightarrow \psi\right) \wedge\left(a_{2}^{2} \rightarrow \psi^{\prime}\right) \wedge\left(a_{3}^{2} \rightarrow \psi^{\prime}\right)$ and cases $\left(\left(a_{1}^{1} \wedge a_{1}^{2}\right) \vee\left(a_{2}^{1} \wedge a_{2}^{2}\right) \vee\left(a_{3}^{1} \wedge a_{3}^{2}\right)\right)$. The cases enfores that the corresponding disjuncts of every disjunct in the group must be simultaneously satisfiable.

We can now start to see where redundancy in dependent disjunctions originates. Because every disjunction in a group of dependent disjunctions must have the same number of disjuncts, some of those disjuncts may appear more than once. In the above example for instance, $\phi$ occurs twice in the first disjunction and $\psi^{\prime}$ occurs twice in the second disjunction. To resolve this problem we impose the following condition, called alternative compactness: if a base constraint $\phi_{j}^{i}$ cquals another base constraint from the same disjunction, $\phi_{k}^{i}$, then the alternatives variables associated with those base constraints, $a_{j}^{i}$ and $a_{k}^{i}$, are also equal. ${ }^{7}$ Doing this allows us to express the alternatives from the example above as $\left(a_{1}^{1} \rightarrow \phi\right) \wedge\left(a_{3}^{1} \rightarrow\right.$ $\left.\phi^{\prime}\right) \wedge\left(a_{1}^{2} \rightarrow \psi\right) \wedge\left(a_{2}^{2} \rightarrow \psi^{\prime}\right)$, and the cases as $\left(\left(a_{1}^{1} \wedge a_{1}^{2}\right) \vee\left(a_{1}^{1} \wedge a_{2}^{2}\right) \vee\left(a_{2}^{1} \wedge a_{2}^{2}\right)\right){ }^{8}$ One advantiage of this is that the number of base constraints that must bo checked during satisfaction can potentially be exponentially reduced.

The next section will show how an alternativecase form for a group of dependent disjunctions can be split into a conjunction of two (or more) equivalent forms, thereby (potentially) exponentially reducing the number of alternative variable interactions that must be checked during satisfaction.

\section{Modularization}

Consider again the example from section 2: $\left\langle{ }_{d} \phi, \phi, \phi^{\prime}, \phi^{\prime}\right\rangle \wedge\left\langle{ }_{d} \psi, \psi^{\prime}, \psi, \psi^{\prime}\right\rangle$. Represented as a compact alternative-case form, the alternatives becomes: $\left(a_{1}^{1} \rightarrow \phi\right) \wedge\left(a_{3}^{1} \rightarrow \phi^{\prime}\right) \wedge\left(a_{1}^{2} \rightarrow \psi\right) \wedge\left(a_{2}^{2} \rightarrow\right.$ $\left.\psi^{\prime}\right)$, with cases: $\left(\left(a_{1}^{1} \wedge a_{1}^{2}\right) \vee\left(a_{1}^{1} \wedge a_{2}^{2}\right) \vee\left(a_{3}^{1} \wedge\right.\right.$ $\left.\left.a_{1}^{2}\right) \vee\left(a_{3}^{1} \wedge a_{2}^{2}\right)\right)$. 'The key to determining that the two disjunctions can be split into different

\footnotetext{
${ }^{7}$ Note that this requires being able to determine equality of the base constraints.

${ }^{8}$ In this example, equivalent alternative variables have been replaced by representatives of their equivalence class. So $a_{2}^{1}$ has been replaced by $a_{1}^{1}$ and $a_{3}^{2}$ has been replaced by $a_{2}^{2}$.
}

groups then involves determining that cases can be split into a conjunction of two smaller cases $\left(a_{1}^{1} \vee a_{3}^{1}\right) \wedge\left(a_{1}^{2} \vee a_{2}^{2}\right)$. If the cases can be split in this manner, wo say the cases (and by extension the group of dependent disjunctions) are independent.

\section{Definition 2 (Independence)}

A case form is independent iff it is equivalent to

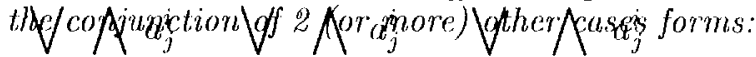
$j \in N i \in M \equiv j \in N^{\prime} i \in M^{\prime} \quad \wedge j \in N^{\prime \prime} i \in M^{\prime \prime}$

where $M^{\prime}$ and $M^{\prime \prime}$ partition $M$

So in the above example, $M=\{1,2\}$ where 1 represents the first disjunction and 2 represents the second. That makes $M^{\prime}=\{1\}$ and $M^{\prime \prime}=\{2\}$. While $M^{\prime}$ and $M^{\prime \prime}$ are clerived from $M$, the clements of the $N$ s are arbitrary. But a consequence of definition 2 is that, $|N|=\left|N^{\prime}\right| \times\left|N^{\prime \prime}\right|$. This will be proved in section 6 . The size of the $N$ s, however, represent the number of cases. So for instance in the above cxample, $N$ might equal $\{1,2,3,4\}$ since there are four disjuncts in the original case form, while $N^{\prime}$ might equal $\{1,2\}$ and $N^{\prime \prime},\{1,2\}$, since the smaller case forms each contain two disjuncts.

The process of splitting a group of dependent disjunctions into smaller groups is called modularization. Modularizing a group of dependent disjunctions amounts to finding a conjunction of case forms that is equivalent to the original case form. The modularization algorithm consists of two main steps. The first is to take the original case form and to construct a pair of possibly independent case forms from it. The second step is to check if these case forms are actually independent from each other with respect to the original one. The modularization algorithm performs both of these steps repeatedly until either a pair of independent case forms is found or until all possible pairs have been checked. If the later, then we know that the original dependent disjunction is already modular. If on the other hand we can split the case forms into a pair of smaller, independent, case forms, then we can again try to modularize each of those, until all groups are modular.

'To construct a pair of potentially independent case forms, wo first need to partition the set of alternative variables from the original case form into two sets. The first subset contains all of and only the variables corresponding to some subset, of the original disjunctions and the second subset of variables is the complement of the first, corresponding to all of and only the other disjunctions. From these subsets of variables, wo construct two new case forms from the original using the operation of confinement, defined below.

\section{Definition $3 a_{j}^{\text {(Confinement) }}$ \\ $\operatorname{conf}\left(j \in N i \in M \quad, M^{\prime}\right)$}




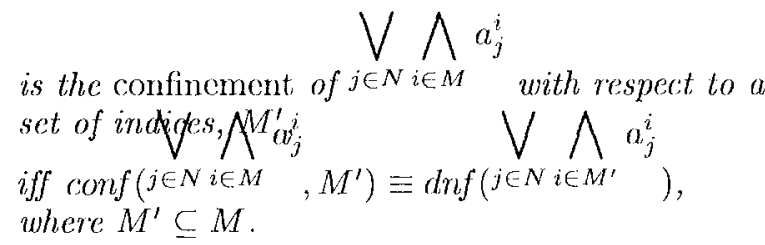

Constructing the confinement of a case form is essentially just throwing out all of the alternative variables that are not in $M^{\prime}$. However, only doing this might leave us with duplicate disjuncts, so converting the result to DNF removes any such duplicates.

To make the definition of confinement clearer, consider the following conjunction of dependent disjunctions:

$\left.{ }_{d} \phi, \phi, \phi, \phi, \phi^{\prime}, \phi^{\prime}\right\rangle \wedge\left\langle{ }_{l} \psi, \psi^{\prime}, \psi, \psi^{\prime}, \psi, \psi^{\prime}\right\rangle \wedge$

$\left\langle a \chi, \chi, \chi^{\prime}, \chi^{\prime}, \chi^{\prime}, \chi^{\prime}\right\rangle$.

This is equivalent to the compact alternative form: ${ }^{9}$

$$
\begin{aligned}
& \left(a_{1}^{1} \rightarrow \phi\right) \wedge\left(a_{5}^{1} \rightarrow \phi^{\prime}\right) \wedge\left(a_{1}^{2} \rightarrow \psi\right) \wedge \\
& \left(a_{2}^{2} \rightarrow \psi^{\prime}\right) \wedge\left(a_{1}^{3} \rightarrow \chi\right) \wedge\left(a_{3}^{3} \rightarrow \chi^{\prime}\right),
\end{aligned}
$$

and the following case form: case $=$

$\left(\left(a_{1}^{1} \wedge a_{1}^{2} \wedge a_{1}^{3}\right) \vee\left(a_{1}^{1} \wedge a_{2}^{2} \wedge a_{1}^{3}\right) \vee\left(a_{1}^{1} \wedge a_{1}^{2} \wedge a_{3}^{3}\right) \vee\right.$ $\left.\left(a_{1}^{1} \wedge a_{2}^{2} \wedge a_{3}^{3}\right) \vee\left(a_{5}^{1} \wedge a_{1}^{2} \wedge a_{3}^{3}\right) \vee\left(a_{5}^{1} \wedge a_{2}^{2} \wedge a_{3}^{3}\right)\right)$.

Now we can compute the confinements. For instance,

$$
\operatorname{conf}(\operatorname{casc},\{1,2\})=\operatorname{dnf}\left(\left(a_{1}^{1} \wedge a_{1}^{2}\right) \vee\left(a_{1}^{1} \wedge a_{2}^{2}\right) \vee\right.
$$$$
\left.\left(a_{1}^{1} \wedge a_{1}^{2}\right) \vee\left(a_{1}^{1} \wedge a_{2}^{2}\right) \vee\left(a_{5}^{1} \wedge a_{1}^{2}\right) \vee\left(a_{5}^{1} \wedge a_{2}^{2}\right)\right) \text {. }
$$

After romoving duplicates we get:

$$
\text { conf }(\text { case },\{1,2\})=
$$$$
\left(\left(a_{1}^{1} \wedge a_{1}^{2}\right) \vee\left(a_{1}^{1} \wedge a_{2}^{2}\right) \vee\left(a_{5}^{1} \wedge a_{1}^{2}\right) \vee\left(a_{5}^{1} \wedge a_{2}^{2}\right)\right) \text {. }
$$

Likewise, for the complement of $M^{\prime}$ with respect to $M$, we get:

$$
\text { conf }(\text { case, }\{3\})=\left(\left(a_{1}^{3}\right) \vee\left(a_{3}^{3}\right)\right) \text {. }
$$

Now we just need to test whether two confined case forms are independent, with respect to the original. This is done with the free combination operation, shown in definition 4.

\section{Definition 4 (Free Combination $\otimes$ )}

The free combination of two case forms is the disjunctive normal form of their conjunction:

case $^{\prime}$ case $^{\prime \prime} \equiv \operatorname{dnf}\left(\right.$ case $^{\prime} \wedge$ case $\left.^{\prime \prime}\right)$

The two case forms, case' and case", are DNF formulae. To compute the free combination, we conjoin them and convert the result back into DNF. They are indepondence if their free combination is equal to the original case form, case.

For cxample, the free combination of the two confinements from above,

$\left(\left(a_{1}^{1} \wedge a_{1}^{2}\right) \vee\left(a_{1}^{1} \wedge a_{2}^{2}\right) \vee\left(a_{5}^{1} \wedge a_{1}^{2}\right) \vee\left(a_{5}^{1} \wedge a_{2}^{2}\right)\right)$ and $\left(\left(a_{1}^{3}\right) \vee\left(a_{3}^{3}\right)\right)$

is

$\left(\left(a_{1}^{1} \wedge a_{1}^{2} \wedge a_{1}^{3}\right) \vee\left(a_{1}^{1} \wedge a_{2}^{2} \wedge a_{1}^{3}\right) \vee\left(a_{5}^{1} \wedge a_{1}^{2} \wedge a_{1}^{3}\right) \vee\right.$ $\left(a_{5}^{1} \wedge a_{2}^{2} \wedge a_{1}^{3}\right) \vee\left(a_{1}^{1} \wedge a_{1}^{2} \wedge a_{3}^{3}\right) \vee\left(a_{1}^{1} \wedge a_{2}^{2} \wedge a_{3}^{3}\right) \vee$ $\left.\left(a_{5}^{1} \wedge a_{1}^{2} \wedge a_{3}^{3}\right) \vee\left(a_{5}^{1} \wedge a_{2}^{2} \wedge a_{3}^{3}\right)\right)$

\footnotetext{
${ }^{9}$ In this example, equivalent altcrnative variables have again been replaced by representatives of their equivalence class. So for instance, $a_{2}^{1}, a_{3}^{1}$ and $a_{4}^{1}$ are all represented by $a_{1}^{1}$.
}

which is not cqual to the original caso form:

$$
\left(\left(a_{1}^{1} \wedge a_{1}^{2} \wedge a_{1}^{3}\right) \vee\left(a_{1}^{1} \wedge a_{2}^{2} \wedge a_{1}^{3}\right) \vee\left(a_{1}^{1} \wedge a_{1}^{2} \wedge a_{3}^{3}\right) \vee\right.
$$

$\left.\left(a_{1}^{1} \wedge a_{2}^{2} \wedge a_{3}^{3}\right) \vee\left(a_{5}^{1} \wedge a_{1}^{2} \wedge a_{3}^{3}\right) \vee\left(a_{5}^{1} \wedge a_{2}^{2} \wedge a_{3}^{3}\right)\right)$, so the first two disjunctions are not independent from the third. Howcver, the second disjunction is independent from the first and the third since conf $($ case, $\{2\})=\left(\left(a_{1}^{2}\right) \vee\left(a_{2}^{2}\right)\right)$, and conf $($ case, $\{1,3\})=\left(\left(a_{1}^{1} \wedge a_{1}^{3}\right) \vee\left(a_{1}^{1} \wedge a_{3}^{3}\right) \vee\left(a_{5}^{1} \wedge a_{3}^{3}\right)\right)$, and their free combination is equal to the original case form. 'Therefore, the original formula is equivalent to $\left\langle d^{\prime} \psi, \psi^{\prime}\right\rangle \wedge\left\langle d^{\prime \prime} \phi, \phi, \phi^{\prime}\right\rangle \wedge\left\langle d^{\prime \prime} \chi, \chi^{\prime}, \chi^{\prime}\right\rangle$.

\section{Free combination elimination}

The last section showed an effective algorithm for modularizing groups of dependent disjunctions. However, even though this is a compile time algorithm we should be concorred about its ofliciency since it has exponential complexity. The main source of complexity is that we might have to check every pair of subsets of disjunctions from the group. In the worst case this is unavoidable (although we do not expect natural language grammars to exhibit, such behavior). Other sources of complexity are computing the freo combination and tosting the rosult against the original caso form. Iuckily it is possible to avoid both of these operations. This can be done by noting that both the original case form and each of the confined case forms are in DNE. 'Therefore it is a necessary condition that if the free combination of the confinements is the same as the original case form then the product of the number of disjuncts in each confinomont, $\mid$ case $^{\prime}|\times|$ case $^{\prime \prime} \mid$, must equal the number of disjuncts in the original case form, |case|. Morcover, since both confinements are derived from the original case form, it, is also a sufficient condition. This is shown more formally in theorem 1.

\section{Theorem 1 (Free combination elimination)}

case $=$ case $^{\prime} \otimes$ case $^{\prime \prime} \Longleftrightarrow \mid$ case $|=|$ case $^{\prime}|\times|$ case $^{\prime \prime} \mid$

Proof $\Rightarrow$ Wo assume that case $\Leftrightarrow$ case $=$ case. Since both case' $\otimes$ case" and case are in DNF and $D N F$ is unique, we know that $\left|\operatorname{case}^{\prime} \operatorname{case}^{\prime \prime}\right|=$ : |case|. We also know that case and case" have no disjuncts in common because they have no alternative variables in common, so $\left|\operatorname{case}^{\prime} \otimes \operatorname{case}^{\prime \prime}\right|=$ $\mid$ case $^{\prime}|\times|$ case $^{\prime \prime} \mid$. Therefore, $\mid$ case $|=|$ case' $\mid \times$ $\mid$ case" $\mid$.

Proof $\Longleftarrow$ Again since case' and case" have no disjuncts in common, we know that |case' $\otimes$ case $^{\prime \prime}|=|$ case $^{\prime}|\times|$ case $^{\prime \prime} \mid$ and thcrefore, that $\mid$ case $|=|$ case $^{\prime} \otimes c^{\prime} a s e^{\prime \prime} \mid$. Every disjunct in case can be represented as $A^{\prime} \wedge A^{\prime \prime}$ where $A^{\prime}$ is a disjunct in case and $A^{\prime \prime}$ is a disjunct in case". So the disjuncts in case $\otimes$ case $^{\prime \prime}$ must be every conjunction of possible $A^{\prime}$ s and $A^{\prime \prime}$ s. So case $\otimes$ case $^{\prime \prime}$ must 
contain all of the disjunctis in cose and it conld

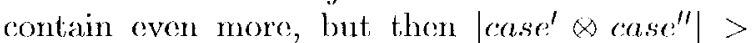
$\mid$ case $\mid$. However, since $\mid$ case $|=-| c a s e^{\prime} \otimes$ case" $\mid$,

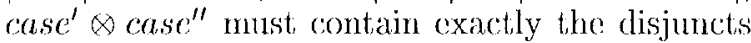
in case and therefore case = case' $\otimes$ case $^{\prime \prime}$.

Wo can see that this would have helped us in the previous example to know that conf (case, $\{1,2\})$ could not be independent from comf $($ aseses, $\{3\})$ with respect to couse becanso $\mid$ conf $($ casse $\{1,2\}) \mid-$ 4 and $\mid$ conf $($ casce, $\{3\}) \mid=2$ hul $\mid$ casce $\mid=6$, not 8. Gonversoly, since $|\operatorname{conf}(\operatorname{cose},\{1,3\})|=3$ and $|\operatorname{com} f(\operatorname{cose},\{2\})|=2$, wo know immediately that these case forms are independent.

This theorem also allows us to perform other combinatorial short culs, such as noting that if the number of disjuncts in the original case form is prime then it is alroady modular.

\section{Conclusion}

this paper has prosented an elficient form for representing dependent disjunctions and an algorithm for detecting and climinating redundant iuteractions within a group of depondent disjumetions. This method shonkl be useful for any system which employs dependent disjunctions since it can eliminate exponontial amounts of processing during constraint satisfaction.

\section{References}

Hiscle, $\Lambda$. and J. Dörre. 1990. Unification of Disjunctive Veature Descriptions IBMM Wissemschaftliches /ontrum, Institute fü̈ Wissensbasierte Systeme tech. report 124.

Gordemann, I). 1991. Parsing and Generation of Unification Grammars. I'hD thesis, U. of Illinois.

Gerdemann, D, and P. King 1994. The Correct and Efficient Implementation of $\Lambda$ ppropriateness Specifications for Typed Foature Structures In COLING 1994, pages 956960.

Griflith, J. 1995. Optimizing leature Structure Unification with 1)ependent D)isjunctions. In l'apers presented at the Workshop on Grammar Formalism for NII' at LSSLILI). U. 'T'ïbingen, Serninar fïr Sprachwissenschaft tech. report, (04-95.

Griffith, J. 1996. Disjunction and Ffficient P'rocessing of leature Structures. Phl) thesis, Universitiät 'Tübingen. Tentalive title.

Maxwoll, J. and R. Kaplan. 1989. An Overview of Disjunctive Constraint Satisfaction. In Proce of Int. Workshop on Parsing Technologies, pages 1827.

Maxwoll, J. and R. Kaplan. 1991. T'ho Interface between Phrassal and linnctional Constraints.
In Constraint Propagation, I,inguistic Description, and Compnetation, Istituto Dalle Molle II)SIA, Iulgano, Switzerland.

Meurers, D. and G. Minnen. 1995. A Computational Treatment of erpse Lexical Rules as Covariation in Lexical Fintriess. In Proce of the 5th Int. Workshop on Natural Language Understanding and Logic: I'rogramming.

Menders, D). and (r. Minnen. 1996. Off-line Con-

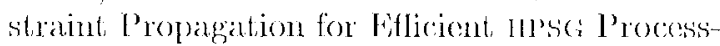
ing. In Proce of IIISG/TAIN-96.

Pollard, C. and 1. Sag. 1994. Head-driven I'hrase. Structure Grammar. C. of Chicago Press.

Strömbäck, L. 1992. Unifying Disjunctive Foature Structures. In COMING 1992, pages $1167-1171$. 